\title{
Low-Code-Plattformen zur Verbreitung von Wearable-Technologien
}

Jannis Vogel und Oliver Thomas

Der Einsatz von Wearable-Technologien wie Smart Glasses wird in Unternehmen durch das spezifische Design und die softwareseitige, individuelle Implementierung erschwert. Für Unternehmen ohne IT-Abteilungen stellt dies eine wesentliche Herausforderung dar. Dies kann ein Projektrisiko bedeuten und schließlich zur Ablehnung der Technologie führen. Insb. betroffen sind klein- und mittelständische Unternehmen, die bedingt durch den Fachkräftemangel und geringere ITBudgets weniger Innovationen in ihrem Unternehmen etablieren können, obwohl sie sich als vorteilhaft für ihre Arbeitsprozesse erweisen. Low-Code-Plattformen, wie sie in diesem Beitrag für Smart-Glasses-basierte Informationssysteme dargestellt werden, überwinden die Implementierungsbarrieren und machen die Technologie für eine Vielzahl an Unternehmen nutzbar.

\section{Einleitung und Motivation}

Die Integration von neuen Technologien in unternehmerischen Abläufen stellt Organisationen vor Herausforderungen. Neben der Identifizierung von nutzenstiftenden Anwendungsfällen ist die Implementierung eine wesentliche Hürde für die Annahme von neuen Technologien. Die Anwendung von Wearable-Technologien wie Smart Glasses ist Gegenstand der aktuellen Forschung und Unternehmen wie DHL (2017), Telekom (Sauter 2017), und Audi (Ubimax 2016) haben ihren Einsatz evaluiert, wie es vergleichend auch im Projekt GLASSHOUSE geschehen ist. Nicht zuletzt sind Innovationsprojekte durch ein entsprechendes Risiko und Investment gekennzeichnet. Insb. kleine und mittlere Unternehmen (KMU) können sich aufgrund des geringen IT-Budgets keine umfangreichen und risikobehafteten Innovationsprojekte leisten (Lindner und Leyh 2019). Die Beschreibung von Anwendungsfällen bzw. sog. Use Cases ist eine weitere Herausforderung bei Innovationsprojekten - Business- und IT-Abteilungen müssen die gleiche „Sprache sprechen“, um fehlerhafte Implementierungen zu vermeiden.

Low-Code-Development ist ein Lösungsansatz, um sowohl eine einfachere Beschreibung von Use Cases als auch eine effektivere Implementierung zu ermöglichen. Das Forschungsinstitut Forrester definiert Low-Code-Plattformen als Platt- 
formen, die eine schnelle, effektive Erstellung von Anwendungssystemen mit möglichst wenig geschriebenen Programmiercode realisieren (Richardson und Rymer 2014). Dabei stützt sich das Prinzip auf das Forschungsfeld der modellgetriebenen Softwareentwicklung. Modelle dienen hierbei zur Beschreibung der Software und zur Erstellung von Anwendungssystemen. Bei der Erstellung wird zwischen der Generierung von Programmiercode und der Interpretation des Modells zum Aufruf bestehender Methoden während der Laufzeit unterschieden (Trompeter et al. 2007; Stahl et al. 2007).

Im Rahmen des GLASSHOUSE-Projekts wurden die Logistikprozesse bei den Anwendungspartnern Meyer \& Meyer sowie Hellmann in der Modellierungssprache Business Process Model and Notation (BPMN) erhoben. Die semi-formalen Modelle dienten zur Beschreibung der aktuellen Ist-Situation und der Herleitung von 36 identifizierten Use Cases innerhalb logistischer Prozesse (Niemöller et al. 2017). Weiterhin wurde die BPMN zur Beschreibung von zukünftigen SmartGlasses-basierten Prozessen verwendet. Auffallend dabei war die unterschiedliche Verwendung von Notationselementen für selbige Smart-Glasses-basierte Funktionalitäten von verschiedenen Modellierungsanwendern (Vogel et al. 2018). Infolgedessen wurde die technologiespezifische Modellierungssprache BPMN4SGA, eine BPMN-Erweiterung für Smart-Glasses-Anwendungen, entworfen und in einem webbasierten Modellierungstool umgesetzt. So können technologische Eigenschaften einer Smart Glasses innerhalb eines Prozesses syntaktisch und semantisch präziser repräsentiert werden (Vogel und Thomas 2019). Alleinstehend repräsentiert BPMN4SGA eine Modellierungssprache, die es ermöglicht, mit BPMN4SGA-Modellen ein gemeinsames Verständnis von Smart-Glasses-basierten Anwendungsfällen zwischen Business- und IT-Abteilungen zu erzielen. Um die zweite beschriebene Problematik der Komplexität der Erstellung von Smart-Glasses-basierten Informationssystemen zu lösen, wurde BPMN4SGA um ein Generierungssystem für Smart-Glasses-basierte Informationssysteme ergänzt.

Im Folgenden beschäftigt sich der vorliegende Beitrag mit den Forschungsfragen: FF1: Wie kann eine Low-Code-Plattform für Smart-Glasses-basierte Informationssysteme gestaltet sein? Und, angeknüpft an die vorherige Forschungsfrage, FF2: Welche Vorteile birgt eine Low-Code-Plattform für Smart-Glasses-basierte Informationssysteme?

Nachstehend werden die wesentlichen Herausforderungen bei der Entwicklung von Smart-Glasses-basierten Informationssystemen, welche die Erstellung einer Low-Code-Plattform für Smart-Glasses-basierte Systeme motivieren (vgl. Abschnitt 2), erläutert. Folgend werden Low-Code-Plattformen für bestehende mobile Informationssysteme und deren Eigenschaften präsentiert (vgl. Abschnitt 3). Die Erkenntnisse werden für eine Low-Code-Plattform für Smart-Glasses-basierte Informationssysteme adaptiert und die integrierte BPMN4SGA wird vorgestellt (vgl. Abschnitt 4). Abschließend folgen ein Ausblick und ein Fazit (vgl. Abschnitt 5). 


\section{Herausforderungen bei der Einführung von Wearables}

Die Einführung von Wearable-Technologien in Unternehmen geht mit mehreren Herausforderungen einher. Zum einen existieren Hardwarerestriktionen, die den Einsatz von Wearables nur unter Einschränkungen bezüglich der Zeit oder der Arbeitsumgebung erlauben. Ein Beispiel dafür ist die Neugestaltung des User Interfaces für kleinere Displays, wie sie bei Smart Watches oder Smart Glasses vorhanden sind. Eine geringere Akkulaufzeit sorgt dafür, dass Wearables nicht eine gesamte Arbeitsschicht von 8 Stunden verwendet werden können, sodass zusätzliche Lademöglichkeiten bedacht werden müssen. Ferner sind Wearables mit einem geringeren Arbeitsspeicher und CPU ausgestattet, sodass dies bei der Gestaltung der Anwendung berücksichtigt werden muss, um einen Absturz der Anwendung zu vermeiden. Schließlich erlauben Wearables neue Interaktionsformen mittels Sprache, Gesten, Touch oder Kontrollknöpfen, die für die Systemsteuerung berücksichtigt werden müssen und entsprechend eines bestmöglichen Bedienkomforts in das System integriert werden sollten (Zobel et al. 2016). Bedingt durch die EU-Datenschutzgrundverordnung (DSGVO) kommen weitere datenschutzrechtliche Anforderungen an die Gestaltung von Informationssystem hinzu (Berkemeier et al. 2017).

Die genannten Limitationen und das Wissen um Gestaltungsrichtlinien für Wearable-Systeme sind wichtig, um die Akzeptanz der Arbeitenden und die Chance auf eine erfolgreiche Integration in die Arbeitswelt zu erhöhen. Hobert und Schumann (2017) identifizierten mit 21 Experteninterviews, dass Unternehmen kaum eigene, erfahrene IT-Experten besitzen, welche die Implementierung von Wearable-Technologien in die unternehmerischen Prozesse und Systeme umsetzen können. Zudem berichten die Befragten, dass es schwierig sei, Softwareanbieter und IT-Berater im Markt zu finden. Ein Interviewpartner hob hervor, dass sein Unternehmen selbst die Software nicht ohne die Hilfe von externen Partnern verändern kann. Damit resultiert aus dem Mangel an IT-Experten eine weitere Barriere bei der Einführung von Wearable-Technologien in Unternehmen.

Nicht zuletzt ergibt sich aus der kontinuierlichen Weiterentwicklung der Wearable-Technologien ein stetiger Anpassungsprozess der Gestaltungsrichtlinien und der Umsetzung der Systeme. Bspw. müssen Gestaltungsprinzipien, die basierend auf der Vuzix M300 Smart Glasses entwickelt worden sind für neue Smart Glasses wie die Vuzix Blade, eine See-Through Glasses, adaptiert werden. Außerdem ermöglichen kommende Technologien wie das 5G-Netz eine neue Gestaltung von Smart-Glasses-basierten Systemen, in denen die Berechnung vermehrt in der Cloud statt auf der Datenbrille stattfindet und durch eine geringere Latenz ermöglicht wird (Sauter 2017). Ergänzt wird dies durch den Trend „Internet of Things“ (IoT), der die Vernetzung von Endgeräten adressiert. Schließlich werden zukünftig Entwicklungen im Bereich der Künstlichen Intelligenz auch in Augmented-Reality-Anwendungen zur Darstellung gewonnener Informationen berücksichtigt. 


\section{Low-Code-Development mittels modellgetriebener Softwareentwicklung}

Es gibt bereits mehrere Anbieter von Low-Code-Plattformen, die sich auf die Erstellung von mobilen Anwendungen für Smartphone- oder webbasierte Applikationen spezialisieren. Nach Forrester sind die führenden Anbieter Microsoft, OutSystems, Mendix, Kony und Salesforce (Rymer und Koplowitz 2019). Dabei zeichnen sich Low-Code-Plattformen insb. durch eine schnellere Entwicklung von Anwendungen aus. Durch die modellbasierte Erstellung der Anwendungen können Business-Systeme flexibel an verändernde Unternehmensanforderungen angepasst werden. Zudem ermöglichen die Modelle eine bessere Verständigung zwischen Business und IT. Schließlich sind Low-Code-Plattformen ein „Enabler“ für Unternehmen mit kleinen IT-Abteilungen, um für sie eigentlich komplexe IT-Projekte umsetzen zu können. Weiterhin begegnen Low-Code-Plattformen dem Fachkräftemangel, indem auch Nicht-Informatiker eine unternehmerische Anwendung umsetzen können. Schließlich ist eine Anwendung, die mit einer Low-Code-Plattform erstellt wurde, oftmals kostengünstiger als ein vergleichbares, notwendiges Softwareprojekt zur Erstellung der Anwendung.

Low-Code-Plattformen sind in der Regel so gestaltet, dass sie vor allem sehr generische Anwendungsfälle, wie eben die Erstellung einer einfachen Smartphone-App, durch die Plattform abdecken, um eine möglichst breite Nutzerbasis anzusprechen. Im Umkehrschluss können Low-Code-Plattformen sehr spezielle Unternehmensanforderungen an eine Anwendung nur bedingt realisieren. Nischenanbieter, bspw. für Wearable-Technologien, sind daher bisher nur kaum oder gar nicht im Markt vertreten.

Schließlich ist die Entwicklung einer Low-Code-Plattform mittels modellgetriebener Softwareentwicklung mit einem erheblichen Aufwand verbunden. Bei der Entwicklung einer Low-Code-Plattform muss zunächst das verfolgte Ziel der Plattform definiert werden. Welche Business-Anwendungen sollen mit der LowCode-Plattform erzeugt werden? Weiterhin ist der Grad der erzeugten Anwendung zu definieren. Soll die Low-Code-Plattform den Anwendern eine komplette Erstellung von Business-Systemen ohne eigenen Programmiercode ermöglichen oder ist eine Möglichkeit zur eigenen Ergänzung von Programmiercode notwendig? Die Repräsentation der Modellierungssprache bzw. die Realisierung des Tools zur Visualisierung von Modellen ist ein entscheidendes Teilsystem einer Low-CodePlattform. Insb. die intuitive Verwendung des Modellierungstools ist entscheidend, um effektiv die Anwendung gestalten und umsetzen zu können. Dabei ist eine zentrale Fragestellung, wie technologische Eigenschaften innerhalb der Modelle repräsentiert werden sollen. Ein weiteres wichtiges Teilsystem ist die Erstellung des Generierungssystems, welches basierend auf den erstellten Modellen eine Anwendung erzeugt. Zusammenfassend ist eine Low-Code-Plattform aufgrund der zwei Teilsysteme des Modellierungstools und des damit verknüpften Generierungssystems äußerst komplex in der Erstellung, Anpassung und Wartung. 


\section{Low-Code-Development für Smart Glasses mittels BPMN4SGA}

\subsection{Anforderungen an die Low-Code-Plattform}

Mit der Einführung von Smart-Glasses-basierten Informationssystemen sind soziale und technische Herausforderungen verbunden. Eine Low-Code-Plattform kann diesen Herausforderungen implementierungsseitig effektiv begegnen, sodass Unternehmen ohne IT-Abteilungen einen möglichst einfachen Zugang zur Technologie und die Implementierung in deren Geschäftsprozessen zu erhalten. Nachfolgend werden Anforderungen an einer solchen Low-Code-Plattform erläutert.

\subsubsection{Cloud-basierter Zugriff}

Ein cloud-basierter Zugriff erlaubt Unternehmen ohne IT-Infrastruktur die Gestaltung und Implementierung von Smart-Glasses-basierten Informationssystemen. Eine Low-Code-Plattform, die auf einer Cloud aufsetzt, hat zudem den Vorteil, dass die Services zur Gestaltung und Umsetzung von Anwendungen einfach für den Anwender zugänglich werden. Zudem kann die Low-Code-Plattform mit der Nutzungslast variabel skalieren. Dies reduziert insg. die IT-Kosten. Daher schließt sich eine On-Premise-Lösung, die der Kunde selbst auf eigenen Servern hosten kann, aus. Der Installations- und Wartungsaufwand wäre zu hoch für die anvisierte Zielgruppe und würde eine zusätzliche Barriere für die anwendenden Unternehmen bedeuten.

\subsubsection{Modellbasierte Repräsentation}

Nach dem abbildungsorientierten Modellverständnis stellt ein Modell eine vereinfachte Darstellung eines Ausschnittes aus der Realität dar (Schütte 1999). Modellierungssprachen wie die ereignisgesteuerte Prozesskette (EPK) oder die BPMN besitzen Notationselemente, deren Verwendung bspw. mit einem Metamodell definiert ist (Object Management Group 2013). Die mit einer Modellierungssprache erzeugten Modelle verfolgen einen Zweck: Die BPMN oder EPK dienen dazu, unternehmerische Geschäftsprozesse abzubilden (Gadatsch 2010). Da diese Prozessmodellierungssprachen keinerlei technologische Konzepte von Smart Glasses berücksichtigen, ist eine neue Modellierungssprache bzw. eine Anpassung einer bestehenden Modellierungssprache notwendig, um Smart-Glasses-basierte Prozessmodelle syntaktisch und semantisch präziser abbilden zu können. Eine möglichst präzise Darstellungsweise ist aus zwei Sichtweisen notwendig. Zum einen brauchen Entwickler ausreichend Informationen und einen gewissen Detailierungsgrad, um basierend auf Modellen ein Smart-Glasses-basiertes System zu implementieren. Zum anderen ist der Detaillierungsgrad notwendig, um technisch eine modellgetriebene Softwareentwicklung zu realisieren. 


\subsubsection{Wiederverwendung von Systemmodulen}

Die Erstellung von Smart-Glasses-basierten Systemen ist bedingt durch neue Konzepte wie Gestenerkennung, Sprachsteuerung oder Objekterkennung komplexer als die Entwicklung von Anwendungen für ein Smartphone. Die softwareseitige Umsetzung dieser Konzepte ist mit personellem Aufwand, welcher Kosten verursacht, verbunden. Daher soll eine technologiespezifische Modellierungssprache insb. die technologischen Konzepte einer Smart Glasses berücksichtigen. In Kombination mit einem Low-Code-Development-Ansatz, können obige Konzepte mittels Systemmodulen vielfach für mehrere Unternehmen wiederverwendet werden. Dabei setzt die Wiederverwendung eine Generalisierung der Systemmodule voraus, welche dadurch spezifisch für den jeweiligen Unternehmenszweck in der Modelldarstellung angepasst werden können. Folglich würden die gesamten ITKosten für die Erstellung der Low-Code-Plattform von mehreren Unternehmen getragen.

\subsubsection{Low-Code-Development}

Unternehmen sollen mit der Low-Code-Plattform für Smart-Glasses-basierte Systeme befähigt werden, für die Bedürfnisse ihrer Unternehmung eigenständig und ohne externe Hilfe Smart-Glasses-basierte Anwendungen gestalten, anpassen und umsetzen zu können. Daher sieht die Low-Code-Plattform den engsten Begriff einer Low-Code-Plattform vor (Richardson und Rymer 2014), die ohne eigenen geschriebenen Programmiercode auskommt. In der modellgetriebenen Softwareentwicklung, die integraler Bestandteil der Low-Code-Plattform ist, wird zwischen Generierung und Interpretation unterschieden. Das Prinzip der Generierung erzeugt basierend auf Modellen Programmiercode, der folgend optional vom Programmierer ergänzt und anschließend kompiliert wird. Im Gegensatz dazu wird bei der Interpretation das zugrundeliegende Modell interpretiert und zur Laufzeit der Anwendung werden Methoden aufgerufen, die die Anwendung dynamisch generiert (Trompeter et al. 2007; Stahl et al. 2007). Da die Kompilierung einer Anwendung, wie einem Android Package (APK-Datei), wie es bspw. auf der Vuzix M300 oder der Google Glass verwendet wird, mit Aufwand für die Einrichtung der Entwicklungsumgebung und die Installation der Anwendung verbunden ist, ist eine weitere Anforderung die Verwendung der modellgetriebenen Softwareentwicklung mittels Interpretation.

\subsection{Beschreibung der Modellierungssprache}

Eine spezifische Modellierungssprache zur Darstellung von Smart-Glasses-basierten Prozessen ist nicht vorhanden. Da Smart Glasses in mobilen Prozessen eingesetzt werden, sind insb. verhaltensorientierte Modellierungssprachen wie die EPK oder BPMN zur Repräsentation von Smart-Glasses-basierten Prozessen geeignet. Bei beiden Prozessmodellierungssprachen fehlt jedoch die Repräsentation von technologischen Konzepten. Aufgrund dessen, dass die BPMN bereits im GLASS- 
HOUSE-Projekt zur Beschreibung von Smart-Glasses-Anwendungsfällen verwendet wurde und die BPMN eine Erweiterungsmöglichkeit auf Metamodellebene besitzt (Group 2013), wurde die BPMN-Erweiterung BPMN4SGA, eine Modellierungssprache für Smart-Glasses-Anwendungen, entwickelt. BPMN4SGA erlaubt die Darstellung von technologischen Eigenschaften einer Smart Glasses innerhalb eines Prozesses. Abb. 1 zeigt die neuen Notationselemente. Jeweils fünf neue Start- und Zwischenereignisse beziehen die technologischen Eigenschaften einer Smart Glasses ein, die einen Einfluss auf die Prozessabfolge und die Darstellung von Informationen haben. Sechs weitere Smart-Glasses-basierte Aktivitäten stellen Aufgaben unter Verwendung der Smart Glasses dar. Bspw. wird die Aktivität Objekterkennung anhand eines Barcodes in einem Warensichtungsprozess verwendet. Zusätzliche Attribute der jeweiligen Aktivitäten und Ereignisse spezifizieren den Prozess (Vogel und Thomas 2019).

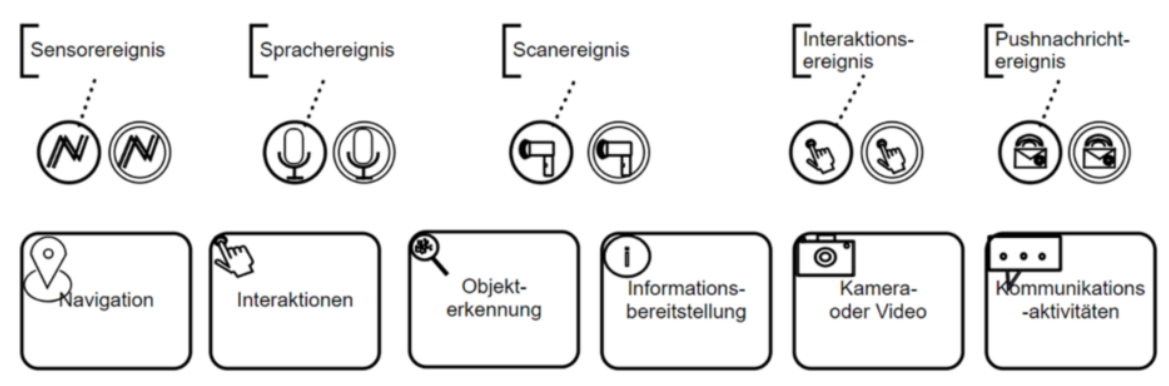

Abb. 1. Notationselemente der BPMN-Erweiterung für Smart Glasses Applications (BPMN4SGA) (Vogel und Thomas 2019)

\subsection{Darstellung der Low-Code-Plattform für Smart-Glasses- basierte Informationssysteme}

Abb. 2 illustriert den Aufbau der Low-Code-Plattform für Smart-Glasses-Anwendungen. Die Plattform besteht aus drei primären Teilsystemen. Das erste Teilsystem integriert unternehmerische Daten und Schnittstellen, wie z.B. zum ERP- oder Kundenmanagementsystem. Ergänzt wird dies um IoT-Daten, die für den Arbeitsprozess relevant sind und weiteren cloud-basierten Services wie Spracherkennung, Datenanalysetools oder Übersetzungssysteme. Außerdem werden die Daten durch ein generiertes BPMN-Log angereichert, welches Prozessinformationen von bereits durchgeführten Smart-Glasses-basierten Prozessen beinhaltet.

Die oben beschriebene Modellierungssprache (vgl. Abb. 1) wurde basierend auf dem webbasierten Modellierungstool BPMN.io (2019) implementiert. Damit die neuen Notationselemente und Attribute syntaktisch und semantisch in die standardisierte BPMN eingebettet sind, wurde das Metamodell zur Beschreibung der BPMN4SGA konzipiert und innerhalb des Tools umgesetzt (Vogel und Thomas 2019). Modellierte BPMN4SGA-Modelle können als XML-Datei heruntergeladen und auf der Plattform gespeichert werden. Damit stehen sie dem freigegebenen 
Benutzer zur Auswahl zur Verfügung. Die Prozesse werden beim Smart-GlassesAnwender manuell oder durch ein Trigger-System ausgelöst. Ein Trigger-System wählt basierend auf vorher definierten Ereignissen, wie bspw. einem Sprachbefehl, einen Prozess aus.

Folgend wird der ausgewählte Prozess interpretiert und spezifische Systemmodule, wie bspw. die Aktivierung der Kamera, Start der Scananwendung oder die Informationsdarstellung einer Liste, werden aufgerufen. Die Aufrufe erfolgen dynamisch anhand der Abfolge der technologischen Notationselemente. Die generierten Daten werden während der Nutzung des Smart-Glasses-basierten Prozesses in einem BPMN-Log gespeichert.

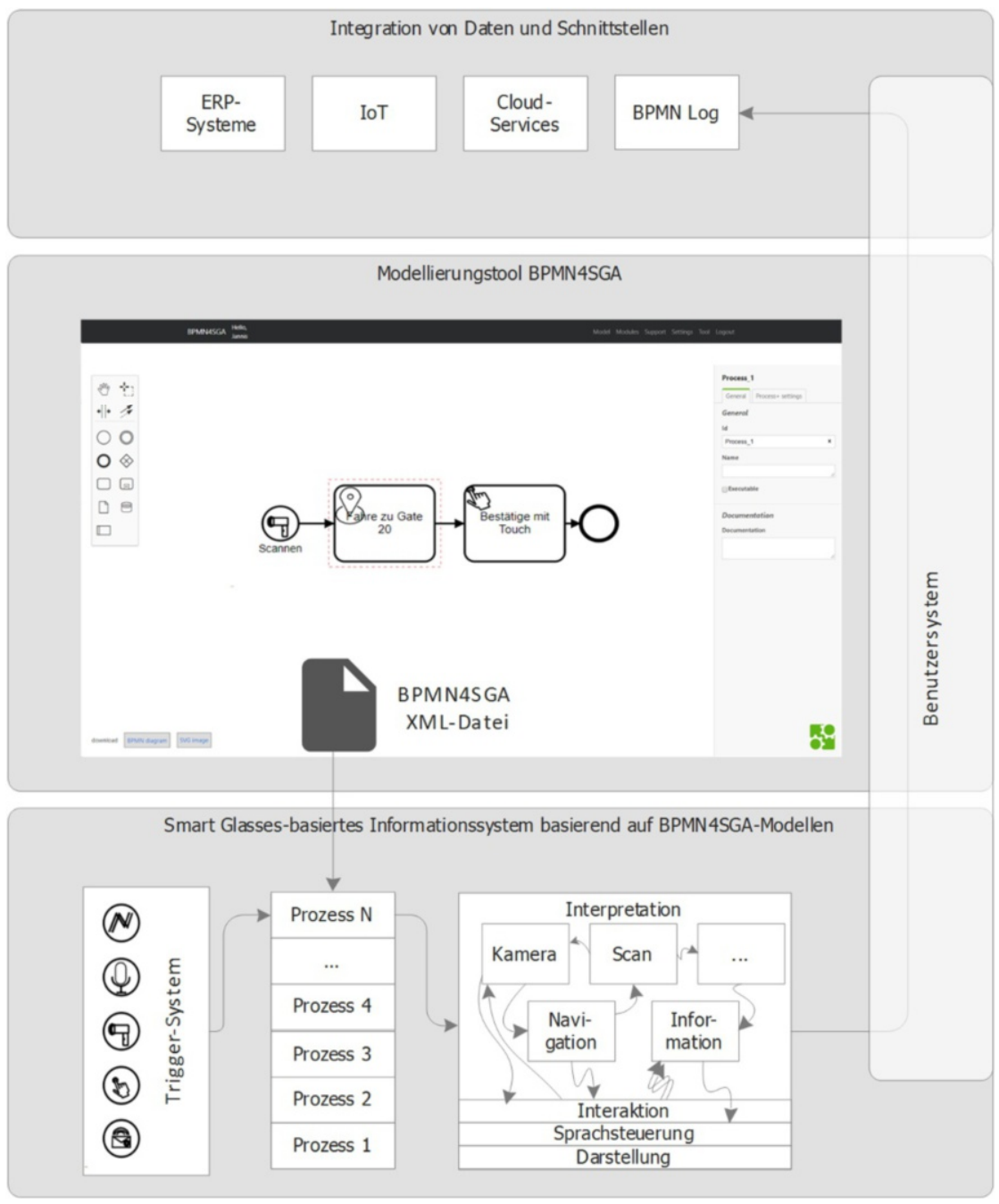

Abb. 2. Aufbau der Low-Code-Plattform für Smart-Glasses-basierte Informationssysteme 


\subsection{Implikationen einer Low-Code-Plattform}

Die prototypische Umsetzung der Low-Code-Plattform für Smart Glasses zeigt, dass sich modellgetriebene Softwareentwicklung auf Wearable-Technologien anwenden lässt. Die Einbettung von technologischen Eigenschaften von Smart Glasses in Prozessmodellen ist aufgrund der Mobilität des Wearables ideal. Dies zeigt unter anderem das von Metzger et al. entwickelte Prozessaufnahme- und Wiedergabesystem mittels Smart Glasses (Metzger et al. 2018). Die entwickelte BPMN4SGA erlaubt eine detaillierte Beschreibung von Smart-Glasses-basierten Anwendungen. In Ergänzung mit einem modellgetriebenen Softwareentwicklungsansatz können Smart-Glasses-basierte Systeme dynamisch generiert werden. Der verfolgte Ansatz erlaubt eine flexiblere und agilere Umsetzung von Smart-Glasses-Anwendungen. Schließlich sind Kosteneinsparungen bei entsprechender Nutzung der Low-Code-Plattform von mehreren Unternehmen zu erwarten, indem die Kosten des Gesamtsystems auf mehrere Akteure verteilt werden können. Ferner resultiert aus der Verwendung einer Low-Code-Plattform eine Veränderung des AufwandNutzen-Verhältnisses von Smart-Glasses-basierten Anwendungsfällen.

Abb. 3 veranschaulicht die Veränderung anhand der identifizierten Use Cases im GLASSHOUSE-Projekt (vgl. Niemöller et al. 2017, eine umfassende Liste der Use Cases kann unter https://www.imwi-data.uni-osnabrueck.de/Business-UseCases.pdf abgerufen werden). Mit einer quantitativen Befragung von 31 Teilnehmern wurde der jeweilige zu erwartende Nutzen eines Use Cases auf einer 7-stufigen Likert-Skala hinsichtlich eines hohen zu erwartenden Nutzens von „1 keinerlei Zustimmung“ bis ,7 einer starken Zustimmung“ erhoben. In einer weiteren Befragung mit 8 Teilnehmern wurden die Befragten hinsichtlich der technischen Realisierbarkeit mittels einer 3-stufigen Likert-Skala von „1 leicht“ bis „,3 schwer“ befragt unter Betrachtung der Faktoren Zeit, Anzahl, Komplexität der Schnittstellen, Komplexität der Algorithmen und Hardwareanforderungen.

Grundsätzlich muss der Nutzen eines Smart-Glasses-basierten Systems größer oder gleich der entstandenen Aufwände sein. Oberhalb der gestrichelten Linie sind Anwendungsfälle, deren Anwendung und Betrieb sich im unternehmerischen Umfeld voraussichtlich rentieren. Die Platzierung der Linie erfolgt unter der Annahme, dass die Implementierung von Smart Glasses in die unternehmerischen Prozesse einen Grundnutzen erzeugt, sodass die gestrichelte Linie grundsätzlich auf der Y-Achse mit dem Wert 4,7 startet. Eine Low-Code-Plattform kann die verursachten Kosten des jeweiligen Systems reduzieren und verschiebt die Systeme, ausgehend unter der Annahme, dass der Nutzen des generierten Systems gleichwertig ist, nach links. In der Grafik erfolgt die weitere Annahme, dass eine LowCode-Plattform für Smart-Glasses-basierte Systeme eine Reduktion von $20 \%$ der hier subjektiv bewerteten Aufwände auf der Skala 1 bis 3 bewirkt und verschiebt die Anwendungsfälle ausgehend von einem gleichbleibenden Nutzen nach links.

Die Low-Code-Plattform fungiert somit als „Enabler“, um vorherig nachteilige Use Cases in einem besseren Aufwand-Nutzen-Verhältnis zu verschieben, sodass vormals nicht umgesetzte Use Cases für Unternehmen finanziell attraktiver wer- 
den. Unter den obigen Annahmen verschiebt sich somit Use Case 6, 11 und 25 durch eine einfachere technische Realisierbarkeit in einem attraktiveren Bereich der Umsetzung, der sich im Allgemeinen in der Grafik in Abb. 3 oben links befindet.

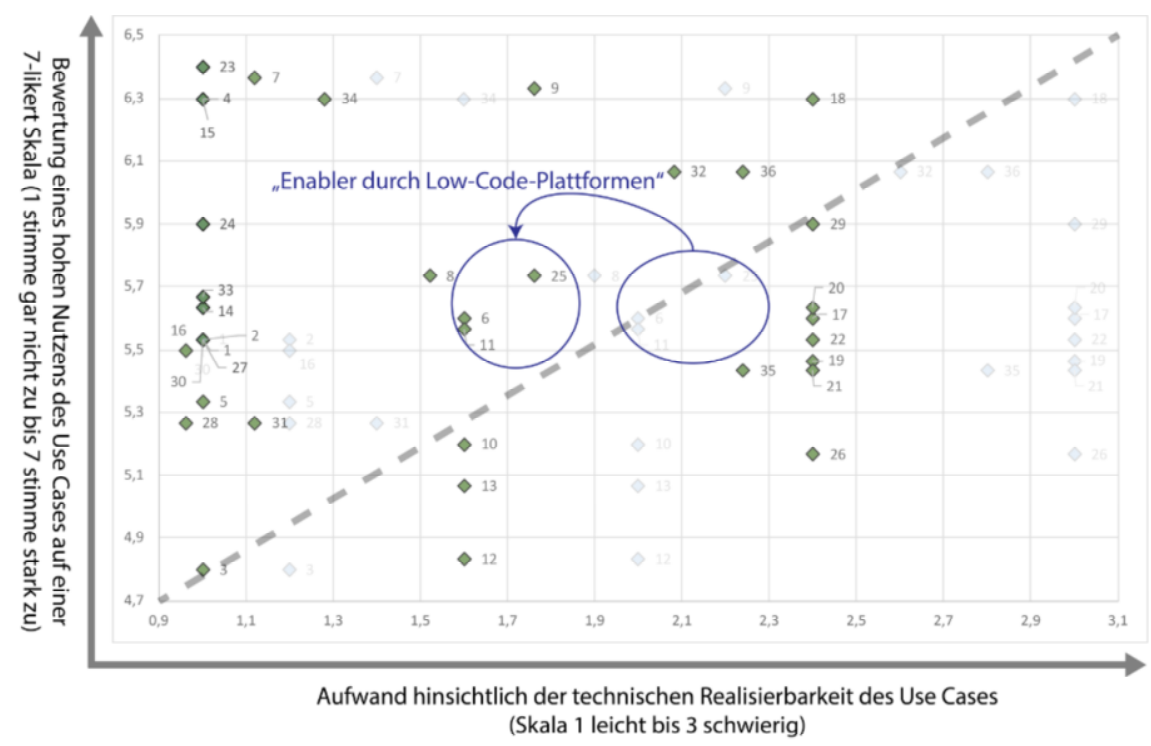

Abb. 3. Modellannahme der Auswirkung einer Low-Code-Plattform für Smart-Glassesbasierte Systeme auf das Aufwand-Nutzen-Verhältnis

Von dieser Entwicklung profitieren insb. kleine und mittelständische Unternehmen, deren IT-Investitionen begrenzt sind und die sich mit einer Low-CodePlattform die Integration von Wearable-Technologien in ihren unternehmerischen Prozessen leisten können. Vorteile sind die finanziell geringeren Einstiegshürden als auch die Realisierung von unternehmensspezifischen Anwendungen ohne ITEntwickler.

\section{Ausblick und Fazit}

Der Beitrag zeigt beispielhaft anhand einer Low-Code-Plattform für Smart-Glasses-basierte Systeme auf, wie Adoptionsbarrieren von Smart Glasses durch eine Kombination aus einer technologiespezifischen Modellierungssprache und einem Generierungssystem für Smart-Glasses-Anwendungen begegnet werden können. Die Low-Code-Plattform adressiert damit zwei wichtige Faktoren bei der Einführung von Wearable-Technologien: Design des Anwendungsfalls und die implementierungstechnische Umsetzung desselbigen. 
Die vorgestellte Low-Code-Plattform für Smart-Glasses-Anwendungen wurde prototypisch umgesetzt, dabei wurden nicht alle Teilsysteme der Plattform in Gänze umgesetzt. Weitere Entwicklungs- und Forschungsarbeiten sind hinsichtlich der technologischen Umsetzung des Trigger-Systems notwendig, bspw. wie bei mehreren auftretenden Ereignissen ein spezieller Prozess ausgewählt wird. Ferner fehlt ein Einbezug der Daten von unternehmerischen Systemen, wie bspw. einem ERP-System. Die umfassende Datenintegration und die Einbettung in BPMN4SGA-Modellen wird als zukünftiger Weiterentwicklungsbedarf gesehen. Als weitere Herausforderung wird der Aufbau von effektiven Low-Code-Plattformen gesehen, die durch einen generischen Aufbau eine Vielzahl an Anwendungsfällen realisieren können, aber dennoch eine Individualisierung ermöglichen, ohne in einem zu komplexen System zu münden.

Hersteller von Wearable-Technologien sollten die Förderung bzw. Erstellung von Low-Code-Plattformen fokussieren, da insb. neue Technologien von Adoptionsbarrieren betroffen sind und diesen effektiv mittels Low-Code-Plattformen begegnet werden kann.

\section{Literatur}

Berkemeier L, McGuire MR, Steinmann S, Niemöller C, Thomas O (2017) Datenschutzrechtliche Anforderungen an Smart-Glasses-basierende Informationssysteme in der Logistik. In: Eibl, Maximilian; Gaedke, Martin (Hrsg) Informatik 2017. Lecture Notes in Informatics (LNI), Gesellschaft für Informatik, Bonn, 1037-1048

BPMN.io (2019) Web-based tooling for BPMN. http://bpmn.io/, abgerufen am 07.06.2019

DHL (2017) Vision Picking bei DHL - Augmented Reality in der Logistik. https://www.dpdhl.com/de/presse/mediathek/videos/dhl-vision-picking-pilotprojekt.html, abgerufen am 25.11.2019

Gadatsch A (2010) Grundkurs Geschäftsprozess-Management. Vieweg + Teubner, Wiesbaden, 6, aktualisierte Auflage

Hobert S, Schumann M (2017) Enabling the Adoption of Wearable Computers in Enterprises - Results of Analyzing Influencing Factors and Challenges in the Industrial Sector. In: Proceedings of the 50th Hawaii International Conference on System Sciences, 4276-4285

Lindner D, Leyh C (2019) Digitalisierung von KMU - Fragestellungen, Handlungsempfehlungen sowie Implikationen für IT-Organisation und IT-Servicemanagement. HMD Praxis der Wirtschaftsinformatik 556(2):402-418

Metzger D, Niemöller C, Jannaber S, Berkemeier L, Brenning L, Thomas O (2018) The Next Generation - Design and Implementation of a Smart Glasses-based Modelling System. Enterprise Modelling and Information Systems Architectures International Journal of Conceptual Modeling 13(18):1-25

Niemöller C, Zobel B, Berkemeier L, Metzger D, Werning S, Adelmeyer T, Ickerott I, Thomas O (2017) Sind Smart Glasses die Zukunft der Digitalisierung von Arbeitsprozessen? Explorative Fallstudien zukünftiger Einsatzszenarien in der Logistik. In: Leimeister J, Marco BW (Hrsg) 13. International Conference on Wirtschaftsinformatik (WI). St. Gallen, 410-424 
Object Management Group (2013) Business Process Model and Notation (BPMN). http://www.omg.org/spec/BPMN/2.0.2/PDF/, abgerufen am 25.11.2019

Richardson C, Rymer JR (2014) New Development Platforms Emerge For CustomerFacing Applications. Forrester Report, https://www.forrester.com/report/New+Development+Platforms+Emerge+For+Custo merFacing+Applications/-/E-RES113411\#, abgerufen am 07.07.2020

Rymer JR, Koplowitz R (2019) The Forrester Wave TM : Low-Code Development Platforms For AD \& D Professionals, Forrester Report Q1 2019, https://www.forrester.com/report/The+Forrester+Wave+LowCode+Development+Plat forms+For+ADD+Professionals+Q1+2019/-/E-RES144387, abgerufen am 07.07.2020

Sauter M (2017) Deutsche Telekom und Zeiss arbeiten an Datenbrille. https://www.golem.de/news/smart-glasses-deutsche-telekom-und-zeiss-arbeiten-andatenbrille, abgerufen am 25.11.2019

Schütte R (1999) Zum Realitätsbezug von Informationsmodellen, EMISA Forum, 26-35

Stahl T, Völter M, Effting S; Haase A (2007) Modellgetriebene Softwareentwickung Techniken, Engineering, Management. dpunkt Verlag, Heidelberg, 2., aktual. Auflage

Trompeter J, Pietrek G, Beltran JCF, Holzer B, Kamann T, Kloss M, Mork SA, Niehues B, Thoms K (2007) Modellgetriebene Softwareentwicklung - MDA und MDSD in der Praxis. entwickler.press, Frankfurt am Main

Ubimax (2016) Audi Smart Factory - Future of Audi Production. https://www.ubimax.com/de/referenzen/audi-casestudy.html, abgerufen am 25.11.2019

Vogel J, Thomas O (2019) Generating Smart Glasses-based Information Systems with BPMN4SGA: A BPMN Extension for Smart Glasses Applications. In: Proceedings 14. International Conference on Wirtschaftsinformatik (WI)., Siegen

Vogel J, Zobel B, Jannaber S, Thomas O (2018) BPMN4SGA: A BPMN Extension for Smart Glasses Applications to enable Process Visualisations. In: Czarnecki C, Brockmann C, Sultanow E, Koschmider A, Selzer A (Hrsg) Workshops der INFORMATIK 2018 - Architekturen, Prozesse, Sicherheit und Nachhaltigkeit. Köllen Druck+Verlag GmbH, Bonn, 259-273

Zobel B, Berkemeier L, Werning S, Thomas O (2016) Augmented Reality am Arbeitsplatz der Zukunft: Ein Usability-Framework für Smart Glasses. In: Mayr HC, Pinzger M (Hrsg) Informatik 2016. Gesellschaft für Informatik e.V., Bonn, 1727-1740 